\title{
Etiología del Autismo: Un Tema a Debate
}

\section{Etiology of Autism: A Subject of Debate}

\author{
Rosa María Rivas Torres \\ Universidad de Santiago de Compostela
}

\author{
Santiago López Gómez \\ Universidad de A Coruña
}

\author{
Eva María Taboada Ares \\ Universidad de Santiago de Compostela
}

\begin{abstract}
Resumen. El autismo es un trastorno de causa desconocida. Sus manifestaciones heterogéneas, junto con la disparidad de criterios utilizados en las investigaciones, dificultan su estudio. Se han propuesto diversos modelos etiológicos que han generado distintas hipótesis. Sin embargo, sigue sin poder ofrecerse una explicación clara y definitiva sobre su génesis. El objetivo de este artículo es valorar los diferentes postulados teóricos que tratan de identificar los mecanismos patogénicos correlacionando las características inconstantes y heterogéneas del autismo con acontecimientos de distinta naturaleza -médicos, genéticos, sociofamiliares, perigestacionales, neurológicos, entre otros-. Al respecto, puede concluirse que la explicación más acertada en la etiología del autismo apunta a una predisposición biológica que proviene del propio entorno y de la constitución personal así como un, todavía no identificado, número de factores tanto de riesgo como protectores.
\end{abstract}

Palabras clave: autismo, etiología, teorías etiológicas.

\begin{abstract}
Autism is a disorder of unknown origin. Its heterogeneous symptoms, together with the disparity of criteria used in its research, make its study difficult. Several etiological models have been proposed, which have generated various hypotheses. However, a clear and definitive explanation about its genesis has not yet proved possible. The aim of this article is to evaluate a variety of theoretical postulates that are intended to identify the pathogenic mechanisms by correlating autism's variable and heterogeneous characteristics with different events (medical, genetic, social, family, perigestational, and neurological events, among others). In this regard, it is concluded that the most adequate explanation for the etiology of autism suggests a biological predisposition originating from both environment and physical condition, together with a number of risk and protection factors that still remain unidentified.
\end{abstract}

Key words: autism, etiology, etiological theories.

\section{Introducción}

A diferencia de otros trastornos neuropsicológicos, identificados desde hace muchos años, el interés por el estudio del autismo no se inicia hasta la publicación del artículo titulado "Alteraciones autísticas de contacto afectivo" (1943), por Leo Kanner.

\footnotetext{
La correspondencia sobre este artículo debe enviarse a la primera autora a la Universidad de Santiago de Compostela, Facultad de Psicología, Dpto. de Psicología Evolutiva y de la Educación. Campus Sur S/N. 15782 Santiago de Compostela.E-mail: rosa.rivas@usc.es
}

En la bibliografía especializada, la definición de autismo y del resto de los trastornos generalizados del desarrollo resulta poco precisa, al igual que su etiología es confusa lo que, con probabilidad, se debe a la falta de nitidez en criterios y protocolos de evaluación. Esta situación dificulta tanto los juicios diagnósticos como la intervención, a lo que se añade una escasa y ambigua participación de estrategias preventivas y de fórmulas basadas en la educación para la salud, que podrían llegar a minimizar 
la alta incidencia del trastorno y sus graves consecuencias.

En la actualidad, el estudio del autismo genera múltiples incógnitas. Uno de los aspectos más confusos en el que se está investigando es el relativo a su etiología. A lo largo de estas últimas décadas, se han propuesto diferentes modelos etiológicos del autismo, considerando sus manifestaciones comportamentales, y/o valorando su diferenciación neurológica e incluso genética. A continuación, se revisan estas teorías, destacando tanto sus puntos fuertes como aquellos otros controvertidos, con el objetivo de establecer una conclusión valorativa del estado del arte sobre esta cuestión.

\section{El trastorno autista}

El autismo se agrupa en torno a los denominados trastornos generalizados del desarrollo que, a su vez, están incluidos en los denominados trastornos de inicio en la infancia, la niñez o la adolescencia (American Psychiatric Association [APA], 2000; Organización Mundial de la Salud [OMS], 1992). Se caracteriza por una perturbación grave y generalizada en varias áreas del desarrollo: i) habilidades para la interacción social, ii) habilidades para la comunicación y iii) la presencia de comportamientos, intereses o actividades estereotipados. Las alteraciones cualitativas son claramente impropias del nivel de desarrollo o edad mental del sujeto. Se expresa durante los primeros años de vida y, frecuentemente se asocia a algún grado de retraso mental, observándose, a veces, en otras enfermedades médicas. La alteración debe manifestarse antes de los 3 años de edad por retraso o funcionamiento anormal en por lo menos una (aunque de manera frecuente varias) de las siguientes áreas: interacción social, lenguaje tal como se utiliza en la comunicación social o juego simbólico o imaginativo. En la mayoría de los casos no se observa ningún período de desarrollo inequívocamente normal, aunque en un $20 \%$ de ellos los padres informan de un desarrollo relativamente normal durante 1 ó 2 años. En estos casos, los padres pueden indicar que el niño adquirió unas cuantas palabras, perdiéndolas a continuación, o pareciendo estancarse evolutivamente. Por definición, si existe un período de desarrollo normal, éste no puede extenderse más allá de los 3 años. El trastorno no se explica mejor por la presencia de un trastorno de Rett o de un trastorno desintegrativo infantil.

Desde los últimos años, se acepta la descripción del autismo como un síndrome de gran complejidad, con múltiples causas y manifestaciones, que agrupa una amplia colección de síntomas inconstantes y heterogéneos, en el sentido de que son raros de observar en distintos individuos. A pesar de los avances en la investigación del trastorno, hoy en día no existe una definición técnicamente aceptable y universalmente compartida del autismo. Esto se debe, en parte, a la dificultad de describir y comprender las profundas y diversas alteraciones que presentan las personas que lo sufren (Rivière, 1993, 1982).

Sin duda alguna, la definición más aceptada es la de la APA (2002). En la reciente revisión de la cuarta edición del Manual Diagnóstico y Estadístico de los Trastornos Mentales (DSM-IV-TR), se afirma que el autismo tiene como características esenciales la presencia de un desarrollo marcadamente anormal o deficiente de la interacción y comunicación sociales junto con un repertorio sumamente restringido de actividades e intereses. Sus manifestaciones varían mucho en función del nivel de desarrollo y de la edad cronológica del sujeto.

Al igual que sucede con su definición, se da también un grado importante de confusión en los criterios diagnósticos del autismo infantil. Ello obedece a su complejidad, a la multiplicidad de variables, a la no especificidad de los síntomas considerados e incluso a los desacuerdos entre los diversos paradigmas de investigación existentes. Por ello, las descripciones actuales del autismo hacen referencia a un grupo heterogéneo de síntomas, sin que sean a su vez específicos del mismo (Artigas, 2001; Etchepareborda, 2001). En la concepción actual del trastorno autista subyace la idea de un síndrome profundamente heterogéneo y con diferencias individuales 
muy marcadas, que se pueden asociar a diversos trastornos. Se acepta, además, que existen muchos retrasos y alteraciones del desarrollo que se acompañan de síntomas característicos del autismo (Gillberg y Billstedt, 2000). De hecho, al estudiar sus síntomas y manifestaciones tipológicas, suele encontrarse que ciertas dimensiones podrían parecer estar cercanas al desarrollo normal del niño, y otros síntomas, ya más propios del espectro autista, y que se identifican con retrasos evidentes (Pry y Guillain, 2002).

Por todas estas razones, cobra fuerza hablar de trastornos del espectro autista como un continuo de formas, que se asocian con una amplia variedad de características, síntomas, factores etiológicos e incluso respuestas frente a los tratamientos (LópezGómez y García, 2007; López-Gómez, Rivas y Taboada, 2009; Rapin, 2002). Es decir, no se trata de un único trastorno con expresiones fijas o dimensiones y síntomas rígidos, aunque se contemple la tipología más clásica de Kanner. Entonces, se hace necesario hablar de "tipos" o niveles de funcionamiento dentro de un contínuum o espectro autístico. Una aproximación más realista a su heterogeneidad y que valore, a su vez, las diferencias observadas en estos sujetos en los niveles de funcionamiento social, lingüístico, en las habilidades no verbales y tanto en el plano cognitivo como comportamental (Stevens et al., 2000; Teunisse, Cools, van-Spaendonck, Aertes y Berger, 2002).

Además de estas dificultades conceptuales y descriptivas, en otras dimensiones de su estudio también se encuentran lagunas, dudas y posicionamientos vertebrados en la disparidad e incluso la contradicción. Esto es lo que sucede con los indicadores de su prevalencia, o al pretender fijar los límites entre los distintos trastornos generalizados del desarrollo y el autismo, o incluso entre el autismo y otros trastornos (Álvarez, 2007; Artigas, 2001; Etchepareborda, 2001).

De igual forma, resulta evidente, la existencia de un incremento poco preciso en las cifras de su prevalencia, de acuerdo con múltiples investigaciones recientes, que lo sitúan entre el 10 y el 20/10.000
(Baker, 2002; Chakrabarti y Fombonne, 2001; Fombonne, 2003; 2005; Gillberg y Wing, 1999; Yeargin-Allsopp et al., 2003). Cuando, además, decrece la prevalencia de retraso mental (Fombonne, 2003). Por ello, ante esta disparidad de tasas, lo más apropiado, en la tendencia actual, es reflejar una prevalencia creciente desde el 2/10.000 al 1/1.000 (Folstein, 1999), que incluso puede llegar a tasas cercanas al $1 \%$ de la población si se considera toda la amplitud de las condiciones del espectro autista (Baird et al., 2006), afectando siempre de manera mayoritaria, en una relación de 4:1, a hombres sobre mujeres (Fombonne, 1999; 2005; Rapin, 1999).

\section{Teorías y modelo patogénicos}

Las evidencias científicas señalan que los síntomas que se encuentran en el espectro autista son el resultado de alteraciones más o menos generalizadas del desarrollo de diversas funciones del sistema nervioso central (Gillberg et al., 1991). Aunque en los últimos años parece cada vez cobrar más sentido el considerar una multiplicidad de factores en la etiología del autismo (Folstein, 1999). Actualmente, la realidad de un mecanismo causal biofisiológico toma fuerza, atendiendo siempre al papel de los factores hereditarios con una compleja y pluridimensional contribución genética (DeLong, 1999; Trottier, Srivastava y Walker, 1999). Todo ello, valorando la interacción entre el potencial genético y una multiplicidad de eventos prenatales y perinatales (LópezGómez, Rivas y Taboada, 2008a, 2008b; Wilkerson, Volpe, Dean y Titus, 2002), puesto que la explicación única de la genética no puede hacer frente a toda la variabilidad manifiesta en el espectro autista ni el resto de los trastornos generalizados del desarrollo. Cabe considerar, al mismo tiempo, ciertos factores del desarrollo (raza, partos múltiples, riesgos paternos) asociados con características demográficas (edad y educación materna, nivel socioeconómico, etc.), interactuando con la vulnerabilidad genética, e incrementando, de este modo, el riesgo 
de autismo (Baron-Cohen y Bolton, 1994; Croen, Grether y Selvin, 2002).

$\mathrm{Al}$ revisar la literatura, se encuentran teorías que destacan la influencia de las interacciones de los padres con el hijo en la patogénesis del trastorno autista. Estas teorías sugieren déficits, relaciones e interacciones alteradas, desde muy temprana edad, que podrían asociarse con la gestación del autismo. Se han ofrecido aproximaciones e hipótesis psicogénicas de corte variado, sobre todo psicodinámico, referidas al autismo, de entre ellas se podrían destacar las de Mahler, Meltzer, Tustin, Misés, entre otros (véase Jiménez, Justo, Louzao, Fernández y Rodríguez, 1987). Sin embargo, esta visión, como refiere Mackowiak (2000), ha cambiado en los últimos años, siendo los factores biológicos los únicos que, hoy en día, parecen mostrarse como causantes de este trastorno, sea a nivel neurológico, bioquímico, genético o por diferentes problemas durante el embarazo, parto o en el desarrollo neonatal. Destacando, como se ha indicado, la heterogeneidad del trastorno y de la inexistencia de un modelo único que explique su etiología (López-Gómez et al., 2009).

El primer grupo de hipótesis a las que se hace referencia incluye a las teorías de corte genetista que cobran cada vez más fuerza al mostrar un amplio soporte empírico (Bayés et al., 2005; Cook, 2001; Folstein, 1999) y sobre todo debido al avance en las técnicas genómicas. Dentro de ellas, los estudios de las ratios hombre/mujer, junto con el riesgo de recurrencia en hermanos, así como los resultados en investigaciones con gemelos indican que los factores genéticos juegan un papel importante (Bailey et al., 1995), principalmente en una alterada regulación en la formación del sistema nervioso en los primeros meses del desarrollo embrionario (Gillberg et $a l ., 1991)$. Se sugiere que son varios los genes que actúan de manera independiente y desconocida para causar el autismo y/o los trastornos generalizados del desarrollo (Bayés et al., 2005; Estecio, Fett, Varella, Fridman y Silva, 2002; Stodgell, Ingram y Hyman, 2001). El riesgo moderado de recurrencia en hermanos y la gran diferencia de concordancia entre gemelos monocigóticos sobre los dicigóticos, con una mayor concordancia en los primeros (Andres, 2002; Bailey et al., 1995), parece indicar, como proponen Bolton et al. (1994), que el sujeto debe heredar varios genes, aun no localizados, para expresar el fenotipo del autismo. Se apunta que el número probable de genes sea entre dos y cuatro, aunque podrían llegar incluso a diez o más (Cook, 2001; Smalley, Arsanouw y Spence, 1991), con su modo complejo y variado de transmisión, lo que genera, asimismo, niveles de afectación y anomalías asociadas diversas (Konstantareas y Homatidis, 1999; Valente, 1997).

En efecto, se han ofrecido diversidad de argumentos sobre varias identificaciones genéticas, entre ellas, sobresalen: HRAS (Comings, Wu, Muhleman y Sverd, 1996); 5HTT (Cook, 2001; Cook et al., 1997); o el HLA (Warren et al., 1996), entre otras posibles. Sin embargo, falta todavía un soporte experimental efectivo en estas teorías. La no identificación del gen o genes específicos que den lugar al autismo, así como al hecho de no haber encontrado los marcadores genéticos ni bioquímicos que lo identifiquen, pese a todos los avances tecnológicos, parece sugerir que se trata de un déficit neuropsicológico que se puede manifestar junto con alteraciones anátomo-funcionales cerebrales e incluso metabólicas. La expresión heterogénea del trastorno, según la cual las lesiones o alteraciones neurológicas en diferentes áreas o estructuras corticales definirían las distintas manifestaciones autísticas, correlaciona con este supuesto.

En los últimos años, se asocia el autismo con una serie de trastornos genéticos y cromosómicos (Gillberg y Billsted, 2000). Destacan, entre ellos, la fenilcetonuria, neurofibromatosis y otros síndromes congénitos (Williams y Hersh, 1998), esclerosis tuberosa (Bolton, Park, Higgins, Griffiths y Pickler, 2002; Gutierrez, Smalley y Tanguay, 1998), y Síndrome de X Frágil (Estecio et al., 2002; Rogers, Wehner y Hagerman, 2001; Turk y Graham, 1997).

Un segundo grupo de hipótesis se centra en el estudio de las alteraciones neuroquímicas y metabólicas vinculadas con los síntomas autistas. Diversos 
estudios (Bailey, Phillips y Rutter, 1996; Sahley y Panksepp, 1987) han tratado de relacionar el exceso de péptidos (de acción similar a los opiáceos) con el comportamiento aislado autista. En este sentido, se han observado grandes mejoras en las manifestaciones clínicas si se bloquean sus efectos (Sandman, Spence y Smith, 1999; Willemsen, Buitellar, vanBerckerlaer y van-Engeland, 1999). Otros estudios identifican un aumento de los niveles de serotonina en sangre como eje fundamental del autismo, y que se asocian con retraso mental y determinados síntomas conductuales del autismo (Aman, Arnold y Armstrong, 1999; Cook, 1990; Leckman y Lombroso, 1998; McDougle, Kresch y Posey, 2000; Strauss, Unis, Cowan, Dawson y Dager 2002). Si bien, otras investigaciones apuntan a que la hiperserotonemia no es real, ni que el ajuste en los niveles de serotonina mejoren realmente los síntomas de los déficits sociales ni comunicativos (Posey, Guenin, Kohn, Swiez y McDougle, 2001). Por lo tanto, continúa sin clarificarse el mecanismo metabólico responsable, al igual que el motivo por el cual cuando se disminuyen los niveles de serotonina, se produce una mejora real en la conducta, aunque no siempre. El DSM-IV-TR (APA, 2002) indica, al respecto, que existen diferencias de grupo en algunas medidas de la actividad serotoninérgica, pero no constituyen un criterio diagnóstico del trastorno autista.

En esta línea, estudios recientes han tratado de relacionar la presencia de altos niveles de testosterona fetal y el autismo. Diversas investigaciones (Auyeung et al., 2009; Baron-Cohen, Lutchmaya y Knickmeyer, 2004) afirman que existe una relación entre el diagnóstico de autismo y su asociación con la testosterona fetal. Si bien, la necesidad de realizar nuevas y amplias investigaciones se hace evidente para poder dar consistencia a estos estudios.

En otra línea, se sitúan las hipótesis de la "teoría de la mente" -TM-, que buscan, en el déficit de la modularidad cognitiva, la causa necesaria del síndrome conductual del autismo (Baron-Cohen, 1998; 2002). La modularidad de la mente es un constructo que sirve, en este caso, para explicar las diversas ejecuciones, desarrollos y variabilidades en los suje- tos autistas, considerando la organización y arquitectura de la mente (Gómez y Núñez, 1998). El enfoque de esta teoría sostiene que la capacidad de atribuir estados mentales a sí mismos y a los demás, como forma de explicar y predecir el comportamiento, no se desarrolla normalmente en los sujetos autistas (Frith, 2002; Frith y Frith, 2003; Pacherie, 1999). Delimita la ausencia de control en los procesos que rigen los estados mentales en el autismo, con su falta de representación simbólica, deseos o capacidad para predecir otros estados mentales, tanto propios como ajenos (Frith, 2002; Hobson, 1995; Martín, Gómez, Cháez y Greer, 2006).

Este hecho se vincula con las alteraciones en la modularidad de la mente y con la manifestación atípica de emociones, al no tener esa capacidad de "leer la mente" con respecto a los deseos y las creencias de los demás (Frith y Frith, 2003; Rieffe, Terwogt y Stockmann, 2000; Tirapu, Pérez, Erekatxo y Pelegrín, 2007). El déficit autista resultaría de una disfunción biológica del SNC y de una organización cortical diferente que, funcionalmente, provocaría un déficit en los mecanismos del aprendizaje. Derivado, todo ello, de alteraciones perinatales durante el desarrollo del sistema nervioso.

La "Asociation International Autisme-Europe" (2000) confirma que las personas incluidas dentro del espectro autista muestran deficiencias para procesar la información. Estas anomalías incluyen trastornos en la regulación de la vigilancia y de diferentes componentes de la atención, al igual que un desarrollo alterado que limita la adecuada percepción y comprensión del mundo, disminuyendo, asimismo, la capacidad para entender los pensamientos, emociones y las intenciones de los demás (Hobson, 1995). Este déficit neurocognitivo es a la vez perceptivo y ejecutivo, si bien, las bases fisiológicas de estos fenómenos no son todavía claras. Aunque esta teoría ha generado múltiples investigaciones, son numerosas también las críticas que se le hacen desde diversas posiciones (Serra, Loth, van Geert, Hurkens y Minderaa, 2002).

Dentro de las teorías de corte neuropsicológico, cabe destacar la "teoría del déficit de las funciones 
ejecutivas" (Ozonoff et al., 1991). Según esta teoría, los déficits que muestran los sujetos autistas se deben a alteraciones en el lóbulo frontal. Concretamente, en el sistema modulador de la activación cortical que provoca un estado crónico de hiperactivación, generando una serie de síntomas similares a los observados en pacientes con lesiones en el lóbulo frontal, e incluso prefrontal (Dawson et al., 2002). Estas lesiones correlacionan con déficits en la atención, cuyo origen puede ser una alteración en los mecanismos de las imágenes motoras. En otras palabras, este enfoque mantiene que la causa fundamental del autismo puede ser una alteración de la función ejecutiva, definida como la capacidad para mantener el "set" adecuado de solución de problemas de cara a la consecución de una meta futura (Pacherie, 1999). Esto afecta a comportamientos tales como la planificación, el control de impulsos, la inhibición de respuestas prepotentes pero irrelevantes, el mantenimiento del "set", la búsqueda organizada y, la flexibilidad del pensamiento y la acción (Ozonoff, Rogers y Pennington, 1993; Tirapu et al, 2007). Entre los síntomas observados en el autismo, de acuerdo con esta alteración cortical frontal, destacan: ausencia de empatía, falta de espontaneidad, afectividad pobre, reacciones emocionales inusuales -hipo e hiper-reactivas-, rutinas, perseveraciones, conducta estereotipada, intereses restringidos, creatividad limitada y, dificultades en la focalización de la atención. Algunos estudios han identificado, mediante técnicas de neuroimagen, estas alteraciones y su relación con la agnosia visual en autistas (Jambaque, Mottron, Ponsot y Chiron, 1998). Aunque otras investigaciones han encontrado más consistente la posibilidad de déficits temporales, sobre todo en la zona del hipocampo, implicados en las disfunciones autísticas (Dawson, Meltzoff, Osterling y Rinaldi, 1998; Hembry, Sánchez y Winslow, 2001). Por otra parte, no se descarta que los déficits en el autismo estén causados por diversas asimetrías cerebrales, tal y como han confirmado diferentes estudios (Harden, Minshem, Millikarjuhn y Keshavan, 2001; Hazlett et al., 2005; Muñoz et al., 2004). No obstante, han ido emergien- do diversas críticas en contra de estas teorías, que plantean, nuevos retos para su investigación (Griffith, Pennington, Wehner y Rogers, 1999).

Dentro de la aproximación neurobiológica al estudio del autismo, se abre una línea de trabajo que estudia la ausencia de reciprocidad emocional en el autismo (Ayuda-Pascual y Martos-Pérez, 2007). En los últimos años, se ha hecho evidente la existencia de una disfunción del sistema de neuronas en espejo como explicativa frente al déficit de procesos de identificación presentes en los autistas y, a su vez, definitorio de la patología observada en los TEA (Dapretto et al., 2006; Iacoboni y Mazziotta, 2007; Oberman et al., 2005; Ramachandran y Oberman, 2006). Así, el sistema de neuronas en espejo podría afectar a las mismas funciones que se observan alteradas en el autismo. Se ha apuntado, asimismo, que estos procesos son necesarios, tanto para desarrollar y controlar nuestros propios movimientos, como para responder también al movimiento de los otros, a partir de respuestas bimodales -visuales y motoras-. Se ha hipotetizado que juegan un papel importante en las habilidades de imitación y en la realización de diversas formas de aprendizaje por imitación, como también para la adquisición del lenguaje, la expresión emocional y la capacidad empática (Cornelio-Nieto, 2009). Su desarrollo inadecuado genera deficiencias de comportamiento que se manifiestan a modo de carencias frente a la capacidad de entender y responder de manera adecuada al comportamiento de los demás (Oberman et al., 2005). La teoría de la disfunción del sistema de neuronas en espejo apoyaría también la teoría de la mente, pues considera la incapacidad de los autistas para acceder a la representación de los estados mentales de los demás, ni a sus emociones, intenciones y motivaciones (Cornelio-Nieto, 2009).

Bajo otra perspectiva, se ha demostrado que existen una serie de problemas asociados al embarazo y el parto que pueden relacionarse con el autismo (Eaton, Mortensen, Thomsen y Frydenberg, 2001). A este respecto, puede afirmarse que determinadas características maternas (como la edad, el peso, el consumo de tabaco y alcohol, la ingesta de medica- 
mentos teratógenos durante el embarazo, etc.), así como ciertos problemas en el parto (infecciones virales: rubéola, citomegalovirus, herpes simplex; rotura prematura de membranas, parto distócico, etc.), se asocian con el autismo (Ghaziuddin, AlKhouri y Ghaziuddin, 2002; López-Gómez et al, 2008a, 2008b; Nelson y Bauman, 2003; Patterson, 2002; Wilkerson et al., 2002). En este sentido, tenemos que hablar de embarazo de riesgo y, consecuentemente, identificar una serie de factores asociados a este riesgo. Los riesgos durante el embarazo no sólo se definen desde el punto de vista médico-obstétrico, sino contextual, psicológico y sociodemográfico (Gray y Dean, 1991). Es evidente que los resultados de un embarazo de riesgo pueden conllevar alteraciones físicas, sean por trastornos congénitos o por complicaciones en el desarrollo del embarazo y/o parto (Koniak y Turner, 2001). Se observan, a su vez, repercusiones psicopatológicas diversas (Arseneault, Tremblay, Boulerie y Saucier, 2002), trastornos psiquiátricos (Eaton et al., 2001), déficits cognitivos (Bratchelor, Dean, Gray y Wenck, 1989), alteraciones comportamentales (Weissman, Warner, Wickramaratne y Kandel, 1999), trastornos generalizados del desarrollo (Wilkerson et al., 2002), dificultades de aprendizaje (Hill, Cawthorne y Dean, 1998) y alteraciones neuropsicológicas (Jones et al., 1998).

La existencia de factores genéticos -anomalías cromosómicas-, los factores maternales -edad, paridad, historial médico, etc.-, los efectos teratógenos de los fármacos administrados a la madre, los factores sociales y demográficos, la prematuridad, los embarazos múltiples, los problemas asociados directamente al feto/neonato -prematuridad, bajo peso, etc.-, son sólo algunos de los factores tras la posibilidad de riesgo en un embarazo que pueden condicionar, no sólo la propia viabilidad fetal, sino también su morbilidad e incluso la de su madre (Gray y Dean, 1991; López-Gómez, Cajal, Ordóñez y Uribe, 2008). La asistencia habitual que, en la actualidad, se ofrece a las embarazadas supone una reducción de las complicaciones asociadas, tal y como reflejan las tasas de morbi-mortalidad perinatal referidas a los últimos años, en comparación con hace sólo unas décadas (MacDorman, Minino, Strobino y Guyer, 2002). Coincide, por otro lado, con un descenso notable de los índices de natalidad y fertilidad en los países desarrollados (Fretts, Schmittdiel, McLean, Usher y Goldman, 1995), pero también con un incremento notable en la prevalencia del autismo (Fombonne, 2003). Esto no significa que, hoy en día, todos los embarazos sigan un proceso favorable, pues surgen, en algunas situaciones, condiciones que lo pueden complicar (Tomashek, Hsia e Iyasy, 2003). Estas situaciones de alto riesgo son responsables de un alto porcentaje de los resultados perinatales adversos, por las que los niños nacidos de estos embarazos tienen un número significativamente mayor de sufrir retrasos y desórdenes en el desarrollo (Wilkerson et al., 2002).

El conocimiento exhaustivo de la influencia de los factores de riesgo, tanto aislada como conjuntamente, va a permitir que se reduzcan todavía más las tasas de incidencia de patologías y problemas perinatales (López-Gómez et al., 2008). Son muchos los estudios clínicos que evidencian un alto porcentaje de niños con una variedad de problemas en el desarrollo después de haber sufrido diversas complicaciones y riesgos perinatales (González y Moya, 1996; Lester et al., 2002). Sobre esta premisa, los niños que han vivido complicaciones durante el desarrollo prenatal, durante el parto, o durante los primeros días del nacimiento, muestran un riesgo considerable frente a trastornos físicos, neuropsicológicos, mentales y comportamentales, las cuales reflejan una serie de complicaciones médicas y psicológicas en su desarrollo. Si bien, en el caso del autismo, sigue sin poder dilucidarse su etiología, ni poder asociarla con alteraciones perinatales. Las respuestas ofrecidas no muestran evidencias claras que permitan identificar factores de riesgo específicos prey perinatales asociados al autismo. Sin embargo, son numerosos los estudios que han informado de una incidencia superior de las complicaciones pre y perinatales en las madres de los sujetos autistas en comparación con los grupos control (Gillberg, 1988; López-Gómez et al., 2008a, 2008b; Matsuishi et al., 
1999). En esta línea, una serie de factores obstétricos se han encontrado asociados al autismo, como el uso de sustancias teratógenas, la edad materna avanzada, los sangrados vaginales, las infecciones virales, la aspiración fetal del meconio, etc. (Tsai y Stewart, 1983; Wilkerson et al., 2002). Aunque también es preciso señalar que, de manera genérica, no se han podido replicar consistentemente muchos de estos estudios y, únicamente, ante factores de riesgo muy evidentes, se han encontrado datos similares entre ciertas investigaciones (Lord, Mulloy, Wendelbe y Schopler, 1991).

Por último, se han desarrollado otras teorías, cuyas hipótesis etiológicas y explicaciones todavía son escasamente abordadas de manera experimental. Ahora bien, no por ello dejan de tener rigor, pues son líneas provechosas de trabajo futuro ante tanta controversia, y sólo la investigación de los próximos años podrá refutarlas o confirmarlas. Desde este planteamiento, se apunta, como causa del autismo, a las radiaciones ambientales, a carencias nutricionales y vitamínicas asociadas a trastornos metabólicos (Page, 2000), o a procesos bioquímicos alterados, que afectan a la digestión y absorción de ciertos alimentos, como el gluten o la caseína (Whiteley et al., 2000), a déficits en el crecimiento cerebral (Courchesne et al., 2001), a la contaminación ambiental, a deficiencias inmunitarias y toxinas patógenas que dañan el cerebro (Burger y Warren, 1998), como las altas concentraciones de proteínas de albúmina y gammaglobulina en sangre (Croonenberghs et al., 2002), a la exposición de determinadas sustancias, como talidomida, pitocina, oxitocina, y otras, durante estadios tempranos de la formación cerebral (Rodier, 2002), a las vacunaciones masivas, y sobre todo a la triple vacuna de sarampión, paperas y rubéola (Madsen et al., 2002; Taylor et al., 2002), o al contenido de thimerosal de las mismas, a la organización disfuncional de los circuitos neuronales (Gustafsson, 1997), entre otras. No obstante, estudios recientes van aportando datos, aunque también poco concluyentes, en contra de algunos de estos riesgos (DeStefano y Chen, 2001; Krause, He, Gershwin, Shoengeld, 2002; Madsen et al., 2003;
Tidmarsh, 2003). Todo ello, sin duda, genera la necesidad de seguir investigando ya no sólo sobre los factores etiológicos de riesgo, sino también en la búsqueda de posibles identificadores y de marcadores biológicos y etiológicos del autismo.

\section{Conclusiones}

El trastorno autista se expresa mediante un conjunto variable y disperso de síntomas que describen un espectro sintomático con perturbaciones graves y generalizadas en varias áreas del desarrollo. Ello explica, en parte, la gran dispersión existente en los criterios diagnósticos.

Las investigaciones actuales sobre el autismo reflejan también su imprecisión a nivel de las explicaciones etiológicas. Existe un cierto grado de consenso en los signos biofisiológicos frente a las explicaciones de tipo funcional. Así, las teorías genéticas muestran una gran fuerza y un amplio soporte empírico. Incluso puede afirmarse que, en un alto número de casos, los factores genéticos están presentes y juegan un papel importante en la etiología del autismo. Se han identificado, además, distintos trastornos genéticos y cromosómicos ligados a diferentes comportamientos autistas.

Las alteraciones neuroquímicas y metabólicas, aunque necesitan más estudios confirmatorios, evidencian la presencia, normalmente durante las etapas prenatales, de diversas sustancias que se asocian con determinados síntomas conductuales del autismo.

Las teorías neuropsicológicas tratan de explicar la alteración en la modularidad de la mente, la ceguera mental y los déficits ejecutivos presentes en los sujetos autistas, identificando las posibles disfunciones del SNC, la presencia de una disfunción en el sistema de neuronas en espejo y de diversos mecanismos neuropsicológicos.

El enfoque centrado en el estudio de los riesgos perigestacionales ofrece escasas evidencias sobre la presencia de riesgos perinatales y el autismo. En este sentido, las respuestas ofrecidas no muestran 
evidencias claras que permitan identificar factores de riesgo específicos pre- y perinatales asociados al autismo.

En definitiva, son muchas las teorías y distintos los estudios que cruzan diversidad de factores y resultados que es preciso reorientar en futuras investigaciones. Tal y como acontece en el estudio de las alteraciones neuropsicológicas, la explicación de un determinado trastorno de esta naturaleza gira en torno a un conjunto heterogéneo de signos y manifestaciones. Ahora bien, de acuerdo con un número importante de investigadores, podría tratarse de distintos trastornos con su correspondiente variabilidad etiológica pero con un resultado clínico similar. Muestra de ello es el cambio en el concepto del trastorno autista en los últimos años, el cual va en la dirección de un "contínuum o espectro autista", que pretende describir toda su dispersión y variabilidad, junto con la imprecisión de los límites entre el autismo y el resto de TGDs e incluso frente a otros trastornos de sintomatología cercana.

En la actualidad, la explicación más acertada e integradora sobre la etiología del autismo debe ser considerada desde una predisposición biológica y un, todavía no identificado, número de factores -genéticos, neuropsicológicos y ambientales- que provocan graves y profundas consecuencias neuropsicológicas, expresadas de manera muy heterogénea.

\section{Referencias}

Álvarez, E. (2007). Trastornos del espectro autista. Revista Mexicana de Pediatría, 74, 269-276.

Aman, M.G., Arnold, L.E. y Armstrong, S.C. (1999). Review of serotonergic agents and perseverative behaviour in patients with developmental disabilities. Mental Retardation and Developmental Disabilities Research Reviews, 5, 279-289.

American Psychiatric Association (APA) (2000). Diagnostic and statistical manual of mental disorders (4th ed., text revision), DSM-IV-TR. Whashington: Author (Trad. Cast. (2002). Ma- nual Diagnóstico y Estadístico de los Trastornos Mentales, Cuarta Edición-Texto Revisado. Barcelona: Massón).

Andres, C. (2002). Molecular genetics and animal models in autistic disorders. Brain Research Bulletin, 57, 109-119.

Arseneault, L., Tremblay, R.E., Boulerie, B. y Saucier, J.F. (2002). Obstetrical complications and violent delinquency: testing two developmental pathways. Child Development, 73, 496-508.

Artigas, J. (2001). Las fronteras del autismo. Revista de Neurología, 2, 211-224.

Association International Autisme Europe (AIAE) (2000). Description de lÁutisme, document prepare sous les auspicies do "Conseil dÁdministration dÁutisme Europe”. Bruselas.

Avyeung, B., Baron-Cohen, S., Ashwin, E., Knickmeyer, R., Taylor, K. y Hackett, G. (2009). Fetal testosterone and autistic traits. British Journal of Psychology, 100, 1-22.

Ayuda-Pascual, R. y Martos-Pérez, J. (2007). Influencia de la percepción social de las emociones en el lenguaje formal de niños con síndrome de Asperger o autismo de alto funcionamiento. Revista de Neurología, 44, 57-59.

Bailey, A., Le Courter, A., Gottesman, I., Bolton, P., Simmonoff, E., Yuzda, E., et al. (1995). Autism as a strongly genetic disorder: evidence from a British twin study. Psychological Medicine, 25, 63-77.

Bailey, A., Phillips, W. y Rutter, M. (1996). Towards an integration of clinical, genetic and neuropsychological perspectives. Journal of Child Psychology and Psychiatry, 37, 89-126.

Baird, G., Simonoff, E., Pickles, A., Chandler, S., Loucas, T., Meldrum, D., et al. (2006). Prevalence of disorders of the autism spectrum in a population cohort of children in South Thames: The Special Needs and Autims Project (SNAP). Lancet, 368, 210-215.

Baker, H.C. (2002). A comparison study of autism spectrum disorder referrals 1997 and 1989. Journal of Autism and Developmental Disorders, $32,121-125$. 
Baron-Cohen, S. (1998). Does the study justify minimalist innate modularity? Learning and Individual Differences, 10, 179-191.

Baron-Cohen, S. (2002). The extreme male brain theory of autism. Trends in Cognitive Sciences, 6 , 248-254.

Baron-Cohen, S. y Bolton, P. (1994). Autism, the facts. New York: Oxford University Press.

Baron-Cohen, S., Lutchmaya, S. y Knickmeyer, R. (2004). Prenatal testosterone in mind. Cambridge, MA: The MIT Press.

Batchelor, E.S., Dean, R.S., Gray, J.W. y Wenck, S. (1989). Classification rates relative risk factors for perinatal events predicting emotional/behavioral disorders in children. Pre- and Perinatal Psychology Journal, 5, 327-341.

Bayés, M., Ramos, J.A., Cormand, B., Hervás, A., Campo, M., Duran, E., et al. (2005). Genotipado a gran escala en la investigación del trastorno del espectro autista y el trastorno por déficit de atención con hiperactividad. Revista de Neurología, 40(Supl. 1), 187-190.

Bolton, P., MacDonald, H., Pickles, A., Bailey, A., Le Courter, A., Sim, C.H., et al. (1994). A case control family history study of autism. Journal of Child Psychology and Psychiatry, 35, 877-900.

Bolton, P., Park, R.J., Higgins, J.N.P., Griffiths, P.D. y Pickles, A. (2002). Neuro-epileptic determinats of autism spectrum disorders in tuberous sclerosis complex. Brain, 125, 1247-1255.

Burger, R.A. Warren, R.P. (1998). Possible immunogenetic basis for autism Mental Retardation and Developmental Disabilities Research Reviews, 4, 137-141.

Chakrabarti, S. y Fombonne, E. (2001). Pervasive developmental disorders in preschool children. Journal of American Medical Association, 285, 3093-3099.

Comings, D.E., Wu, S.J., Muhleman, D. y Sverd, J. (1996). Studies of the c-Harvey-Ras gene in psychiatric disorders. Psychiatric Research, 63, 2532.

Cook, E. (1990). Autism: review of neurochemical investigation. Synapse, 6, 292-308.
Cook, E. (2001). Genetics of autism. Child and Adolescent Psychiatric Clinics of North America, 10, 333-350.

Cook, E., Courscherne, R., Lord, C., Cox, N.J., Yan, S., Lincoln, A. y Haas, R. (1997). Evidence of linkage between the serotonin transporter and autistic disorder. Molecular Psychiatry, 2, 247250.

Cornelio-Nieto, J.O. (2009). Autismo infantil y neuronas en espejo. Revista de Neurología, 48 (Supl. 2), 27-29.

Courchesne, E., Karns, C. M., Davis, H. R., Ziccardi, R., Carper, R.A., Tigue, Z.D., et al. (2001). Unusual brain growth patterns in early life in patients with autistic disorder: An MRI study. Neurology, 57, 245-254.

Croen, L.A., Grether, J.K. y Selvein, S. (2002). Descriptive epidemiology of autism in a California population: Who is at risk? Journal of Autism and Developmental Disorders, 32, 217224.

Croonenberghs, J., Wauters, A., Devreese, K., Verkerk, R., Scharpe, S., Bosmans, E., et al. (2002). Increased serum albumin, gammaglobin, immunoglobulin $\mathrm{IgG}$, and $\mathrm{IgG} 2$ and $\mathrm{IgG} 4$ in autism. Psychological Medicine, 32, 1457-1463.

Dapretto, M., Davies, M.S., Pfeifer, J.H., Scout, A.A., Sigman, M., Bookheimer, S.Y. y Jacoboni, M. (2006). Understanding emotions in others: mirror neuron dysfunction in children with autism spectrum disorders. Nature Neuroscience. Brief Communications, 9, 28-30.

Dawson, G., Meltzoff, A. N., Osterling, J. y Rinaldi, J. (1998). Neuropsychological correlates of early symptoms of autism. Child Development, 69, 1276-1285.

Dawson, G., Munson, J., Estes, A., Osterling, J., McPartland, J., Toth, K., et al. (2002). Neurocognitive function and joint attention ability in young children with autism spectrum disorder versus developmental delay. Child Development, 73, 345-358.

DeLong, G.R. (1999). Autism: New data suggest a new hypothesis. Neurology, 52, 911-916. 
DeStefano, F. y Chen, R.T. (2001). Autism and measles-mumps-rubella vaccination: Controversy laid to rest? CNS Drugs, 15, 831-837.

Eaton, W.W., Mortensen, P.B., Thomsen, P.H. y Frydenberg, M. (2001). Obstetric complications and risk for severe psychopathology in childhood. Journal of Autism and Developmental Disorders, 31, 279-285.

Estecio, M., Fett, A., Varella, M., Fridman, C. y Silva, E. (2002). Molecular and cytogenetic analyses on Brazilian youths with pervasive developmental disorders. Journal of Autism and Developmental Disorders, 32, 35-41.

Etchepareborda, M.C. (2001). Perfiles neurocognitivos del espectro autista. Revista de Neurología Clínica, 2, 175-192.

Folstein, S. E. (1999). Autism. International Review of Psychiatry, 11, 269-277.

Fombonne, E. (1999). The epidemiology of autism: A review. Psychological Medicine, 29, 769786.

Fombonne, E. (2003). The prevalence of autism. Journal of American Medical Association, 289, 87-89.

Fombonne, E. (2005). The changing epidemiology of autism. Journal of Applied Research in Intellectual Disabilities, 18, 281-294.

Fretts, R., Schmittdiel, J., McLean, F., Usher, R. y Goldman, M. (1995). Increased maternal age and the risk of fetal death. New England Journal Medicine, 15, 953-957.

Frith, U. (2002). Mind blindness and the brain in autism. Neuron, 32, 969-979.

Frith, U. y Frith, C.D. (2003). Development and neurophysiology of mentalizing. Philosophical Transactions of the Royal Society B Biological Sciences, 358, 459-473.

Ghaziuddin, M., Al-Khouri, I. y Ghaziuddin, N. (2002). Autistic symptoms following herpes encephalitis. European Child and Adolescent Psychiatry, 11, 142-146.

Gillberg, C, Steffenburg, S.,Walhstrom, J., Gillberg, I.C., Sjostedt, A., Martinsson, T., et al. (1991). Autism assosiated with marker chromosome.
Journal American Academy Child and Adolescent Psychiatry, 304, 325-329.

Gillberg, C. (1988). The neurobiology of infantile autism. Journal of Child Psychology and Psychiatry, 29, 257-266.

Gillberg, C. y Billstedt, E. (2000). Autism and Aperger syndrome: coexistence with other clinical disorders. Acta Psychiatrica Scandinavica, 102, 321-330.

Gillberg, C. y Wing, L. (1999). Autism: Not an extremely rare disorders. Acta Psychiatrica Scandinavica, 99, 399-406.

Gómez, J. y Nuñez, M. (1998). La mente social y la mente física: desarrollo y dominios de conocimiento. Infancia y Aprendizaje, 84, 5-32.

González, J. y Moya, M. (1996). Asfixia perinatal, encefalopatía hipóxico-isquémica y secuelas neurológicas en recién nacidos a término. II. Descripción e interrelaciones. Revista de Neurología, 24, 969-976.

Gray, J.W. y Dean, R.S. (1991). Behavioral implications of perinatal complications: An overview. En J.W. Gray y R.S. Dean (Eds.), Neuropsychology of perinatal complications (pp. 1-21). New York: Springer Publishing Company.

Griffith, E. M., Pennington, B. F., Wehner, E. A. y Rogers, S. J. (1999). Executive functions in young children with autism. Child Development, 70, 817-832.

Gustafsson, L. (1997). Inadequate cortical feature maps: A neural circuit theory of autism. Biological Psychiatry, 42, 1138-1147.

Gutierrez, G.C., Smalley, S.L. y Tanguay, P.E. (1998). Autism in tuberous sclerosis complex. Journal of Autism and Developmental Disorders, 28, 97-103.

Harden, A.Y., Minshew, N.J., Mallikarjuhn, M. y Keshavan, M.S. (2001). Brain volume in autism. Journal of Child Neurology, 16, 421-424.

Hazlett, H.C., Peo, M., Gerig, G., Smith, R.G., Provenzale, J., Ross, A., et al. (2005). Magnetic resonance imaging and head circumference study of brain size in autism. Archives of General Psychiatry, 62, 1366-1376. 
Hemby, S.E., Sanchez, M.M. y Winslow, J.T. (2001). Functional genomics approaches to a primate model of autistic symptomology. Journal of Autism and Developmental Disorders, 31(6), 551-555.

Hill, S.K., Cawthorne, V. y Dean, R.S. (1998). Utility of the Maternal Perinatal Scale (MPS) in distinguishing normal form learning disabled children. International Journal of Neuroscience, 95, 141-154.

Hobson, R.F. (1995). El autismo y el desarrollo de la mente. Madrid: Alianza.

Iacoboni, M. y Mazziotta, J.C. (2007). Mirror neurons system: basic findings and clinical applications. Annals of Neurology, 62. Mirror neurons system: basic findings and clinical applications. Annals of Neurology, 62, 213-218.

Jambaque, I., Mottron, L, Ponsot, G. y Chiron, C. (1998). Autism and visual agnosia in a child with right occipital lobectomy. Journal of Neurology, Neurosurgery and Psychiatry, 65, 555-560.

Jiménez, C.L., Justo, $\mathbf{M}^{\mathrm{a}}$.A., Louzao, $\mathbf{M}^{\mathrm{a}}$. X., Fernández, M.G. y Rodríguez, A.Mª (1987). As voces de mármore. Autismo e psicoses infantís en Galicia. Vigo: Xerais.

Jones, N.A., Field, T., Fox, N.A., Davalos, M., Lundy, B. y Hart, S. (1998). Newborns of mothers with depressive symtoms are physiologically less developed. Infant Behavior and Development, 21 537-541.

Kanner, L. (1943). Autistic disturbances of affective contact. Nervous Child, 2, 217-250 (Reeditado por L. Kanner (1983). Childhood psychosis: Initial studies and new insigts. New York: Wiley.

Koniak, D. y Turner, C. (2001). Health risks and psychosocial outcomes of early chilbering: A review of the literature. Journal of Perinatal and Neonatal Nursing, 15, 1-17.

Konstantareas, M.M. y Homatidis, S. (1999). Chromosimal abnormalities in a series of children with autistic disorder. Journal of Autism and Developmental Disorders, 29, 275-285.

Krause, I, He, X., Gershwin, M.E. y Shoenfeld, Y. (2002). Brief report: Immune factors in autism: A critical review. Journal of Autism and Developmental Disorders, 32, 337-345.

Leckman, J.F. y Lombroso, P.L. (1998) Development of the cerebral cortex: IV. Transcription factors. Journal American Academy Child and Adolescent Psychiatry, 37, 451-452.

Lester, B.M., Tronick, E.Z. , LaGasse, L., Seifer, R., Bauer, C.R., Shankaran, S., et al. (2002). The maternal lifestyle study: Effects of substance exposure during pregnancy on neurodevelopmental outcome in 1-month-old infants. Pediatrics, 110, 1182-1192.

López Gómez, S., Rivas Torres, R.M. y Taboada Ares, E.M. (2009). Revisiones sobre el autismo. Revista Latinoamericana de Psicología, 41, 555570.

López-Gómez, S. y García, C. (2007). Patrones comportamentales en el trastorno autista: descripción e intervención. Psicología Educativa, 13, 117-131.

López-Gómez, S., Cajal, C.J., Ordóñez, S.M. y Uribe, A.F. (2008). Identificación y valoración neuropsicológica del riesgo perinatal: instrumentos. Revista Colombiana de Obstetricia y Ginecología, 59, 316-326.

López-Gómez, S., Rivas, R.M. y Taboada, E.M. (2008a). Los riesgos maternos pre-peri y neonatales en una muestra de madres de hijos con trastorno generalizado del desarrollo. Psicothema, 20, 684-690.

López-Gómez, S., Rivas, R.M. y Taboada, E.M. (2008b). Detección de los riesgos maternos perinatales en los trastornos generalizados del desarrollo. Salud Mental, 31, 371-379.

Lord, C., Mulloy, C., Wendelboe, M. y Schopler, E. (1991). Pre- and perinatal factors in high-funtioning females and males with autism. Journal of Autism and Developmental Disorders, 21, 197-209.

MacDorman, M. F., Minino, A.M., Strobino, D.M. y Guyer, B. (2002). Annual Summary of vital statistics, 2001. Pediatrics, 110, 1037-1052.

Mackowiak, M.A (2000). Etiology of autism-Focus on the biological perspective. Early Child Development and Care, 160, 77-84. 
Madsen, K. M., Lauritsen, M. B., Pedersen, C. B., Thorsen, P., Plesner, A.M., Anderson, P.H., et al. (2003). Thimerosal and the occurrence of autism: negative ecological evidence form danish population-based data. Pediatrics, 112, 604-606.

Madsen, K.M., Hviid, A., Vestergaard, M., Schendel, D., Wohlfahrt, J., Thorsen, P., et al. (2002). A population-based study of measles, mumps, and rubella vaccination and autism. New England Journal Medicine, 347, 1477-1482.

Martín, M.J., Gómez, I., Chávez, M., y Greer, D. (2006). Toma de perspectiva y teoría de la mente: aspectos conceptuales y empíricos. Una propuesta complementaria y pragmática. Salud Mental, 29, 5-14.

Matsuishi, T., Yamashita, Y., Ohtani, Y., Ornitz, E., Kuriya, N., Murakami, Y., et al. (1999). Brief report: Incidence of and the risk factors for autistic disorders in neonatal intensive care unit suvivors. Journal of Autism and Developmental Disorders, 29, 161-166.

McDougle, C.J., Kresch, L.E. y Posey, D.J. (2000). Repetitive thoughts and behavior in pervasive developmental disorders: Treatment with serotonin reuptake inhibitors. Journal of Autism and Developmental Disorders, 30, 427-435.

Muñoz, J.A., Palau, M., Salvadó, B., Amo, C., Fernández, A., Maestú, F., et al. (2004). Estudio mediante magnetoencefalografía de los trastornos generalizados del desarrollo. Nueva propuesta de clasificación. Revista de Neurología, 38(Supl. 1), 28-32.

Nelson, K. B. y Bauman, M. (2003). Thimerosal and autism? Pediatrics, 111, 674-679.

Oberman, L.M., Hubbard, E.M., McCleery, J.P., Altschuler, E.L., Ramachandran, V.S. y Pineda, J.A. (2005). EEG evidence for mirror neuron dysfunction in autism spectrum disorders. Brain Research. Cognitive Brain Research, 24, 190198.

Organización Mundial de la Salud (OMS - WHO) (1992). The ICD-10 Classification of Mental and Behavioural Disorders: Diagnostic criteria for research. OMS (Trad. Cast. (1992), CIE-10.
Trastornos Mentales y del Comportamiento. Descripciones clínicas y pautas para el diagnóstico. $10^{\mathrm{a}}$ Rev. Madrid: Meditor.

Ozonoff, S., Pennington, B.F. y Rogers, S.J. (1991). Executive function deficits in high functioning autistic individuals: relationship to theory of mind. Journal of Child Psychology and Psychiatry, 32, 108-115.

Ozonoff, S., Rogers, S.J. y Pennington, B.F. (1993). Can standard measures identify subclinical markers of autism? Journal of Autism and Developmental Disorders, 23, 429-441.

Pacherie, E. (1999). Imágenes motoras, autoconciencia y autismo. En J. Russell (Ed.), El autismo como trastorno de la función ejecutiva (pp. 205243). Madrid: Médica Panamericana.

Page, T. (2000). Metabolic approaches to the treatment of autism spectrum disorders. Journal of Autism and Developmental Disorders, 30, 463469.

Patterson, P.H. (2002). Maternal infection: Window on neuroimmune interactions in fetal brain development and mental illness. Current Opinion in Neurobiology, 12, 115-118.

Posey, D.J., Guenin, K.D., Kohn, A., Swiezy, N.B., McDougle, C.J. (2001). A naturalistic open-label study of mirtazapine in autistic and other pervasive developmental disorders. Journal Child and Adolescent Psychopharmacology, 11, 267-277.

Pry, R. y Guillain, A. (2002). Symptomatologie autistique et niveaux de developpement. Enfance, 54), 51-62.

Ramachandran, V.S. y Oberman, L.M. (2006). Broken mirrors: a theory of autism. Scientific American, 295, 62-69.

Rapin, I. (1999). Autism in search of a home in the brain (editorial). Neurology, 52, 902-904.

Rapin, I. (2002). The autistic-spectrum disorders. New England Journal Medicine, 347, 302-303.

Rieffe, C., Terwogt, M.M. y Stockmann, L. (2000). Understanding atypical emotions among children with autism. Journal of Autism and Developmental Disorders, 30 , 195-203.

Rivière, A. (1982). Principios de definición y trata- 
miento del autismo infantil. Boletín del Real Patronato de Prevención y atención a personas con Minusvalías, 5, 7-18.

Rivière, A. (1993). El desarrollo y la educación del niño autista. En A. Marchesi, C. Coll y J. Palacios (Comps.), Desarrollo psicológico y educación, III. Necesidades educativas especiales y aprendizaje escolar (pp. 313-333). Madrid: Alianza.

Rodier, P.M. (2002). Converging evidence from stem injury in autism. Developmental Psychopathology, 14, 537-557.

Rogers, S.J., Wehner, E.A. y Hagerman, R. (2001). The behavioral phenotype in fragile $\mathrm{X}$ : Symptoms of autism in very young children with fragile $\mathrm{X}$ syndrome, idiopathic autism, and other developmental disorders. Journal of Developmental and Behavioral Pediatrics, 22, 409-217.

Sahley, T.L. y Panksepp, I. (1987). Brain opioids and autism: An updated analysis of possible linkcages. Journal of Autism and Developmental Disorders, 17, 201-216.

Sandman, C.A., Spence, M.A. y Smith, M. (1999). Proopiomelanocortin (POMC) disregulation and response to opiate blockers. Mental Retardation and Developmental Disabilities Research Reviews, 5, 314-321.

Serra, M., Loth, F.L., van Geert, P.L. C., Hurkens, E. y Minderaa, R.B. (2002). Theory of mind in children with "lesser variants" of autism: A longitudinal study. Journal of Child Psychology and Psychiatry and Allied Disciplines, 43, 885-900.

Smalley, S.L., Arsanouw, R F. y Spence, M.A. (1988). Autism and genetics. Archives of General Psychiatry, 45, 953-961.

Stevens, M.C., Fein, D. A., Dunn, M., Allen, D., Waterhouse, L.H., Feinstein, C., et al. (2000). Subgroups of children with autism cluster analysis: A longitudinal examination. Journal of American Academy of Child and Adolescent Psychiatry, 39, 346-352.

Stodgell, C.J., Ingram, J.I. y Hyman, S.L. (2001). The role of candidate genes in unravelling the genetics of autism. International Review of Research in Mental Retardation, 23, 57-81.
Strauss, W.L., Unis, A.S. Cowan, C., Dawson, G. y Dager, S. (2002). Fluorine magnetic resonance in pediatric patients treated for pervasive developmental disorders. American Journal of Psychiatry, 159, 755-760.

Taylor, B., Miller, R., Lingan, R., Andrews, N., Simmons, A., Stowe, J., et al. (2002). Measles, mumps and rubella vaccination and bowel problems or developmental regression in children with autism: Population study. British Medical Journal, 324, 393-396.

Teunisse, J.P., Cools, A.R., van-Spaendonck, K.P.M., Aertes, F., Berger, H.J.C., et al. (2002). Cognitive styles in high-functioning adolescent with autistic disorder. Journal of Autism and Developmental Disorders, 31, 55-66.

Tidmarsh, L. (2003). There is little evidence that combined vaccination against measles, mumps, and rubella is associated with autism. EvidenceBased Mental Health, 6, 62.

Tirapu, J., Pérez, G., Erekatxo, M., y Pelegrín ,V. (2007). ¿Qué es la teoría de la mente? Revista de Neurología, 44, 479-489.

Tomashek, K. M., Hsia, J. y Iyasu, S. (2003). Trends in postneonatal mortality atrributable to injury, United States, 1988-1998. Pediatrics, 111, 12191225 .

Trottier, G., Srivastava, L. y Walker, C.D. (1999). Etiology of infantile autism: A review of recent advances in genetic and neurobiological research. Journal of Psychiatry and Neuroscience, 24, 103115 .

Tsai, L. y Steward, M.A. (1983). Etiological implications of maternal age and birth order in infantile autism. Journal of Autism and Developmental Disorders, 13, 57-65.

Turk, J. y Graham, P. (1997). Fragile X syndrome, autism and autistic features. Autism, 1, 175-197.

Valente, M. (1997). Autism: symptomatic and idiopathic and mental retardation. Pediatrics, 48, 495-496.

Warren, R., Singh, V., Averett, R., Odell, J., Maciulis, A., Burger, R. y Daniels, W. (1996). Inmunogenetics studies in autism and related dis- 
orders. Molecular Chemical Neuropathology, 28, 77-81.

Weissman, M.M., Warner, V., Wickramaratne, P.J. y Kandel, D.B. (1999). Maternal smoking during pregnancy and psychopathology in offspring followed to adulthood. Journal American Academy Child and Adolescent Psychiatry, 38, 892-899.

Whiteley, P., Rodgers, J. y Shattock, P. (2000). Feeding Patterns in Autism. Autism, 4, 207-211

Wilkerson, D.S., Volpe, A.G., Dean, R.S. y Titus, J.B. (2002). Perinatal complications as predictors of infantile autism. International Journal of Neuroscience, 112, 1085-1098.

Willemsen, S., Buitellar, J.K., van-Berckerlaer, O. y
van-Engeland, H. (1999). Six months continuation treatment in naltrexone-responsive children with autism: An open-label case-control design. Journal of Autism and Developmental Disorders, 29, 167-169.

Williams, P.G. y Hersh, J.H. (1998). The association of neurofibromatosis type 1 and autism. Journal of Autism and Developmental Disorders, 28, 567571.

Yeargin-Allsopp, M., Rice, C., Karapurkar, T., Doerbberg, N., Boyle, C., Murphy, C., et al. (2003). Prevalence of autism in a US metropolitan area. Journal of American Medical Association, 289, 49-55.

Artículo recibido: 09/07/2009

Revisión recibida: 05/08/2009

Artículo aceptado: 15/08/2009 